\title{
Special function form exact solutions for Jeffery fluid: an application of power law kernel
}

\author{
Maryam Asgir ${ }^{1}$, A.A. Zafar ${ }^{1}$, Abdullah M. Alsharif², Muhammad Bilal Riaz ${ }^{3,4^{*}}$ and Muhammad Abbas $5^{*}$ (D)
}

\section{"Correspondence:}

bilalsehole@gmail.com;

muhammad.abbas@uos.edu.pk

${ }^{3}$ Department of Mathematics,

University of Management and Technology, 40100 C-II Johar Town

Lahore, Pakistan

${ }^{5}$ Department of Mathematics,

University of Sargodha, 40100

Sargodha, Pakistan

Full list of author information is

available at the end of the article

\section{Springer}

\begin{abstract}
This research note's objective is to elaborate on the study of the unsteady MHD natural convective flow of the Jeffery fluid with the fractional derivative model. The fluid flow phenomenon happens between two vertical parallel plates immersed in a porous medium. The one plate is moving with the time-dependent velocity $U_{0} f(t)$, while the other is fixed. The mathematical model is presented with the system of the partial differential equation along with physical conditions. Appropriate dimensionless variables are employed in the system of equations, and then this dimensionless model is transformed into the Caputo fractional-order model and solved analytically by the Laplace transform. The exact expressions for velocity and temperature, which satisfy the imposed initial and boundary conditions, are obtained. Memory effects in the fluid are observed which the classical model fails to elaborate. Interesting results are revealed from the investigation of emerging parameters as Grashof number, Prandtl number, relaxation time parameter, Jeffery fluid parameter, Hartmann number, porosity, and fractional parameter. The results are elucidated with the detailed discussion and the assistance of the graphs. For the sake of validation of results, the corresponding solutions for viscous fluids are also obtained and compared with the solutions already existing in the literature.
\end{abstract}

Keywords: Special functions; Jeffery fluid; Free convection; MHD; Laplace transform; Porous medium; Caputo derivative

\section{Introduction}

The interest developing in the studies of non-Newtonian fluid in the last few years is owing to its practical implementation in industry and technology. Some of the common examples of the non-Newtonian fluid are honey, polymer solution, gel, blood, macro-molecules solutions, and many others. The nonlinear rheological properties of the non-Newtonian fluid are one of the major aspects of its importance [1-3]. Many rheological problem applications are observed in the field of geophysics, bioscience, cosmetics, drying of paper, food processing, chemical plastic production, petroleum industries, and many more. In literature, many models have been suggested to explicate the rheological conduct of nonNewtonian fluids $[4,5]$. In the amidst, Jeffery fluid is noted as an important model of non-Newtonian fluid because of its simplicity. The Jeffery fluid best explains the rheo-

(c) The Author(s) 2021. This article is licensed under a Creative Commons Attribution 4.0 International License, which permits use, sharing, adaptation, distribution and reproduction in any medium or format, as long as you give appropriate credit to the original author(s) and the source, provide a link to the Creative Commons licence, and indicate if changes were made. The images or other third party material in this article are included in the article's Creative Commons licence, unless indicated otherwise in a credit line to the material. If material is not included in the article's Creative Commons licence and your intended use is not permitted by statutory regulation or exceeds the permitted use, you will need to obtain permission directly from the copyright holder. To view a copy of this licence, visit http://creativecommons.org/licenses/by/4.0/. 
logical viscoelastic fluids because it utilizes the time derivative preferably to convected derivative. The linear viscoelastic behavior of Jeffery fluid makes it more appealing in the polymer industries. An important role of Jeffery fluid is observed in blood flow and fluid mechanics due to its viscoelastic behavior. Newtonian fluid can be derived as a special case of Jeffery fluid as it is a significant generalization of a Newtonian fluid. Several studies of the Jeffery fluid flow under different conditions have been carried out by many researchers. Hussain et al. [6] explored the effects of thermal radiation on an MHD incompressible Jeffery nano-fluid past a stretching sheet. Hayat et al. [7] employed the homotopy analysis method (HAM) on the time-dependent mixed convective flow of the Jeffery fluid and reported thermal radiation effects on it. Idowu et al. [8] analyzed the unsteady MHD oscillatory flow of the Jeffery fluid along with the chemical reaction and noted the impacts of heat and mass transfer. Zin et al. [9] explored the thermal radiation impacts on the free convective Jeffery fluid flow along with ramped wall temperature. The analysis of magnetic dipole effects on the convective Jeffery fluid flow past a permeable plate along suction and injection has been performed by Zeeshan et al. [10]. Some more significant studies on the fluid phenomenon can be traced in [11-19] and the references therein.

The study of noninteger-order derivatives and integrals is called fractional calculus, which is a generalization of classical calculus. It is an important and fruitful tool which proved its place by controlling and managing the many physical processes in the field of science and engineering such as electromagnetism, nanotechnology, drilling of oil and water, electrochemistry, and heat transfer processes. The property of fractional derivative to describe the memory effects and nonlocal distribution effects makes them helpful in better understanding and description of the complex phenomena. One of the widely used fractional derivatives is Caputo. Shahid [20] wrote a report on the transference of heat and mass in an MHD flow past over an oscillating plate with the Caputo derivative. The concept of Caputo derivative was implemented by Vieru et al. [21] to reveal the interesting results of the natural convective flow of the generalized viscous fluid. Casson fluid model study with the Caputo derivative was done by Khan et al. [22]. The examination of MHD impacts on the transfer of heat of an Oldroyd-B fluid via nonlocal kernels was carried by Riaz [23]. They explored the semi-analytical solutions, and the results were demonstrated by graphs. Fourier transform was implemented to the proposed study of slip effects on the oscillatory flows of a fractionalized Jeffery fluid in a permeable medium by Khan [24]. An investigation was carried out on the time-dependent free convective flow of the Jeffery fluid past an upright plate via the Laplace integral transform by [25]. The Laplace transform was employed to investigate the analytical solution of the telegraph equation via fractional approach by [26]. Nazish et al. [27] performed the analysis of natural convective fluid flow on the inclined magnetic field. They used the fractional Caputo, Caputo-Fabrizio, and Atangana-Baleanu operators to highlight the influence of transference of heat and mass on fluid flow. Further studies regarding the application of fractional derivatives can be found in the literature and references therein [28-36].

The problem of three-dimensional MHD flow of the Jeffery fluid along Newtonian heating was illustrated by Shehzad et al. [37]. Zafar et al. [38] published an article on the analysis of composite fractional relaxation differential equation via a different fractional operator. The exact solution was attained in special functions form. A novel approach of frac- 
tional derivatives was employed to study the heat and mass movement of MHD Oldroyd-B fluid along with ramped wall velocity and temperature by [39]. The MHD flow of the Jeffery fluid in a channel was explored by Hayat et al. [40], and the series solutions were attained. The study of impacts of MHD on Jeffery fluid via fractional derivative was carried out by various researchers in [41-45].

Motivated by the above, the objective of this manuscript is to study the heat transfer analysis of the MHD fractional Jeffery fluid in a channel with generalized boundary conditions. The Laplace integral transform has been applied to obtain the exact solutions to the problem. The solutions are presented in the series form. The note is comprised of five sections. Section 2 presents the mathematical model and geometry of the problem. The Caputo fractional differential equations and solution of temperature and velocity are explored in Sect. 3. Limiting cases are discussed in Sect. 4. Graphical illustrations of the influence of the pertinent parameters are presented along with interesting results elaboration in Sect. 5. Closing remarks are stated in Sect. 6.

\section{Problem description}

The study of the incompressible, unsteady natural convective flow of MHD Jeffery fluid is elaborated here. The Jeffery fluid is immersed in a porous medium between two vertical plates at $\chi^{*}=0$ and $\chi^{*}=d$. Initially, both plates and fluid are static with the ambient temperature $\theta_{\infty}$. As time increase, the acceleration of the plate at $\chi^{*}=0$ starts with $U_{0} f\left(t^{*}\right)$, keeping the other plate fixed. The plate temperature drops or rises to $\theta_{d}+\left(\theta_{w}-\theta_{d}\right) g\left(t^{*}\right)$, and $f\left(t^{*}\right)$ and $g\left(t^{*}\right)$ are continuous functions that have zero value at $t^{*}=0$. A transverse magnetic force is applied perpendicular to the surface of the plate which acts perpendicular to the fluid flow. Assuming that the velocity is the function of $\chi^{*}$ and $t^{*}$ only, neglecting the induced magnetic field, viscous dissipation, Joule heating effect, and using Boussinesq's approximation are shown in Fig. 1, the governing equations for the fluid flow are [46]

$$
\frac{\partial w\left(\chi^{*}, t^{*}\right)}{\partial t^{*}}=\frac{v}{1+\lambda_{1}}\left(1+\lambda_{r} \frac{\partial}{\partial t^{*}}\right) \frac{\partial^{2} w\left(\chi^{*}, t^{*}\right)}{\partial \chi^{* 2}}
$$

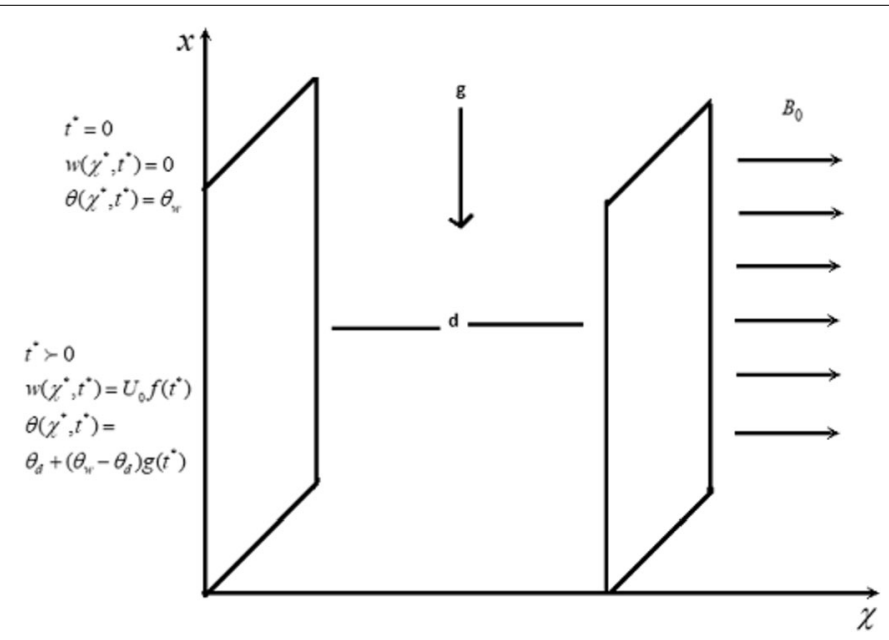

Figure 1 Model geometry with description 


$$
\begin{aligned}
- & \left(\frac{v \phi}{k_{1}}+\frac{\sigma B_{0}^{2}}{\rho}\right) w\left(\chi^{*}, t^{*}\right)+g \beta\left(\theta-\theta_{d}\right), \\
\rho C_{p} \frac{\partial \theta\left(\chi^{*}, t^{*}\right)}{\partial t^{*}} & =k \frac{\partial^{2} \theta\left(\chi^{*}, t^{*}\right)}{\partial \chi^{* 2}},
\end{aligned}
$$

along the following initial and boundary conditions:

$$
\begin{aligned}
& w\left(\chi^{*}, 0\right)=0, \quad \frac{\partial w\left(\chi^{*}, 0\right)}{\partial t^{*}}=0, \quad \theta\left(\chi^{*}, 0\right)=0, \\
& w\left(0, t^{*}\right)=U_{0} f\left(t^{*}\right), \quad w\left(d, t^{*}\right)=0, \\
& \left.\theta\left(0, t^{*}\right)=\theta_{d}+g\left(t^{*}\right)\left(\theta_{w}-\theta_{d}\right)\right), \quad \theta\left(d, t^{*}\right)=\theta_{w} .
\end{aligned}
$$

The dimensionless quantities relations are defined as follows:

$$
\begin{aligned}
w^{\prime} & =\frac{w}{U_{0}}, \quad y^{\prime}=\frac{y^{*}}{d}, \quad \psi^{\prime}=\frac{\theta-\theta_{d}}{\theta_{W}-\theta_{d}}, \quad t^{\prime}=\frac{v t^{*}}{d^{2}}, \\
P_{r} & =\frac{\mu c_{p}}{k}, \quad K=\frac{k_{1}}{\phi d^{2}}, \quad \lambda=\frac{\lambda_{r} v}{d^{2}}, \quad H_{a}^{2}=\sqrt{\frac{\sigma}{\mu}} B_{0} d, \\
G_{r} & =\frac{g \beta \nu\left(\theta_{w}-\theta_{d}\right)}{U_{0}^{3}} .
\end{aligned}
$$

The system of partial differential equations after implementing Eq. (6) into Eqs. (1)-(5) is

$$
\begin{aligned}
& \frac{\partial w(\chi, t)}{\partial t}=\frac{1}{1+\lambda_{1}}\left(1+\lambda \frac{\partial}{\partial t}\right) \frac{\partial^{2} w(\chi, t)}{\partial \chi^{2}}-\left(\frac{1}{K}+H_{a}^{2}\right) w(\chi, t)+G_{r} \psi(\chi, t), \\
& P_{r} \frac{\partial \psi(\chi, t)}{\partial t}=\frac{\partial^{2} \psi(\chi, t)}{\partial \chi^{2}} .
\end{aligned}
$$

The associated initial and boundary conditions change into

$$
\begin{aligned}
& w(\chi, 0)=0, \quad \frac{\partial w(\chi, 0)}{\partial t}=0, \quad \psi(\chi, 0)=0, \\
& w(0, t)=f(t), \quad w(1, t)=0, \quad \psi(0, t)=g(t), \quad \psi(1, t)=1 .
\end{aligned}
$$

\section{Caputo fractional formulation and solutions}

The noninteger-order Caputo fractional derivative operator called $C$ derivative [47] is defined as

$$
{ }^{C} D_{t}^{\eta} h(\varsigma, t)=\frac{1}{\Gamma(1-\eta)} \int_{0}^{t}(t-\vartheta)^{-\eta} h^{\prime}(\varsigma, \vartheta) d \vartheta, \quad \eta \in(0,1) .
$$

By implementing the Laplace transform on the $\mathrm{C}$ derivative, we get

$$
\mathcal{L}\left({ }^{C} D_{t}^{\eta} h(\varsigma, t)\right)=s^{\eta} \mathcal{L}(h(\varsigma, t))-s^{\eta-1} h(\varsigma, 0) .
$$

\subsection{Exact solution of temperature}

In this section, we find the exact solution of temperature by using the Laplace transformation. 
Theorem 1 Let $\mathcal{L}$ be the Laplace operator. Applying this operator on Eq. (8) along with initial and boundary conditions (9) and (10), the exact solution of temperature is

$$
\psi(\chi, t)=\int_{0}^{t} g^{\prime}(t-\tau) \psi_{1}(\chi, \tau) d \tau+\psi_{2}(\chi, t)
$$

where $\psi_{1}(\chi, t)$ and $\psi_{2}(\chi, t)$ are solution functions.

Proof By replacing the time derivative with C derivative operator into Eq. (8), and then applying the Laplace transform, we get

$$
\frac{\partial^{2} \bar{\psi}(\chi, q)}{\partial \chi^{2}}-P_{r} q^{\alpha} \bar{\psi}(\chi, q)=0,
$$

where $\bar{\psi}(\chi, q)$ satisfies the conditions

$$
\bar{\psi}(0, q)=G(q), \quad \bar{\psi}(1, q)=\frac{1}{q} .
$$

The above differential equation solution by using the corresponding boundary conditions Eq. (14) is

$$
\begin{aligned}
\bar{\psi}(\chi, q) & =q G(q)\left(\frac{\sinh \left((1-\chi) \sqrt{P_{r} q^{\alpha}}\right)}{q \sinh \left(\sqrt{P_{r} q^{\alpha}}\right)}\right)+\left(\frac{\sinh \left(\chi \sqrt{P_{r} q^{\alpha}}\right)}{q \sinh \left(\sqrt{P_{r} q^{\alpha}}\right)}\right) \\
& =g G(q) \psi_{1}(\chi, q)+\psi_{2}(\chi, q) .
\end{aligned}
$$

Now consider

$$
\begin{aligned}
\psi_{1}(\chi, q) & =\frac{e^{(1-\chi) \sqrt{P_{r} q^{\alpha}}}-e^{-(1-\chi) \sqrt{P_{r} q^{\alpha}}}}{q\left(e^{\sqrt{P_{r} q^{\alpha}}}-e^{-\sqrt{P_{r} q^{\alpha}}}\right)} \\
& =\sum_{k=0}^{\infty}\left(\frac{e^{-(2 k+\chi) \sqrt{P_{r} q^{\alpha}}}}{q}-\frac{e^{-(2 k+2-\chi) \sqrt{P_{r} q^{\alpha}}}}{q}\right) .
\end{aligned}
$$

Employing the inverse Laplace transform gives the expression of $\psi_{1}(\chi, t)$

$$
\begin{aligned}
\psi_{1}(\chi, t)= & \sum_{k=0}^{\infty}\left(\sum_{n=0}^{\infty} \frac{(-1)^{n}(2 k+\chi)^{n}\left(P_{r}\right)^{n / 2}}{n ! \Gamma\left(1-\frac{n \alpha}{2}\right)} t^{\frac{-n \alpha}{2}}\right. \\
& \left.-\sum_{n=0}^{\infty} \frac{(-1)^{n}(2 k+2-\chi)^{n}\left(P_{r}\right)^{n / 2}}{n ! \Gamma\left(1-\frac{n \alpha}{2}\right)} t^{\frac{-n \alpha}{2}}\right) .
\end{aligned}
$$

Consider now

$$
\begin{aligned}
\psi_{2}(\chi, q) & =\frac{e^{\chi \sqrt{P_{r} q^{\alpha}}}-e^{-\chi \sqrt{P_{r} q^{\alpha}}}}{q\left(e^{\sqrt{P_{r} q^{\alpha}}}-e^{-\sqrt{P_{r} q^{\alpha}}}\right)} \\
& =\sum_{k=0}^{\infty}\left(\frac{e^{-(2 k+1-\chi) \sqrt{P_{r} q^{\alpha}}}}{q}-\frac{e^{-(2 k+1+\chi) \sqrt{P_{r} q^{\alpha}}}}{q}\right),
\end{aligned}
$$


and implementing the inverse Laplace transform on the above equation, we get

$$
\begin{aligned}
\psi_{2}(\chi, t)= & \sum_{k=0}^{\infty}\left(\sum_{n=0}^{\infty} \frac{(-1)^{n}(2 k+1-\psi)^{n}\left(P_{r}\right)^{n / 2}}{n ! \Gamma\left(1-\frac{n \alpha}{2}\right)} t^{\frac{-n \alpha}{2}}\right. \\
& \left.-\sum_{n=0}^{\infty} \frac{(-1)^{n}(2 k+1+\psi)^{n}\left(P_{r}\right)^{n / 2}}{n ! \Gamma\left(1-\frac{n \alpha}{2}\right)} t^{\frac{-n \alpha}{2}}\right) .
\end{aligned}
$$

Employing the inverse Laplace transform on Eq. (15), we have

$$
\psi(\chi, t)=\int_{0}^{t} g^{\prime}(t-\tau) \psi_{1}(\chi, \tau) d \tau+\psi_{2}(\chi, t)
$$

where $\psi_{1}(\chi, t)$ and $\psi_{2}(\chi, t)$ are expressed in Eq. (17) and Eq. (19) respectively.

\subsection{Exact solution of velocity}

In this section, we find the exact solution of velocity by using the Laplace transformation.

Theorem 2 Let $\mathcal{L}$ be the Laplace operator. Applying this operator on Eq. (7) along with initial and boundary conditions (9) and (10), the exact solution of velocity is

$$
w(\chi, t)=I_{1}(\chi, t)+I_{2}(\chi, t)-I_{3}(\chi, t),
$$

where $I_{1}(\chi, t), I_{2}(\chi, t)$, and $I_{3}(\chi, t)$ are solution functions.

Proof Replacing the time derivative with C derivative operator into Eq. (7) and applying the Laplace transform, we get

$$
\begin{aligned}
& \left(\frac{1+\lambda q^{\alpha}}{1+\lambda_{1}}\right) \frac{\partial^{2} \bar{w}(\chi, q)}{\partial \chi^{2}}-\left(q^{\alpha}+\frac{1}{K}+H_{a}^{2}\right) \bar{w}(\chi, q)=-G_{r} \bar{\psi}(\chi, q), \\
& \frac{\partial^{2} \bar{w}(\chi, q)}{\partial \chi^{2}}-\Omega_{2} \bar{w}(\chi, q)=\frac{-G_{r}}{\Omega_{0}} \bar{\psi}(\chi, q),
\end{aligned}
$$

where $\Omega_{0}=\frac{1+\lambda q^{\alpha}}{1+\lambda_{1}}, \Omega_{1}=q^{\alpha}+\frac{1}{K}+H_{a}^{2}$, and $\Omega_{2}=\frac{\Omega_{1}}{\Omega_{0}}$.

The Laplace transformed boundary conditions are

$$
\bar{w}(0, q)=F(q), \quad \bar{w}(1, q)=0 .
$$

The solution of Eq. (22) by embedding Eq. (15) in it and then using Eq. (23) gives

$$
\begin{aligned}
\bar{w}(\chi, q)= & \frac{F(q)}{\sinh \sqrt{\Omega_{2}}} \sinh \left((1-\chi) \sqrt{\Omega_{2}}\right) \\
& +\frac{G_{r}}{q \Omega_{0}\left(P_{r} q^{\alpha}-\Omega_{2}\right) \sinh \sqrt{\Omega_{2}}}\left(q G(q) \sinh \left((1-\chi) \sqrt{\Omega_{2}}\right)-\sinh \left(\chi \sqrt{\Omega_{2}}\right)\right) \\
& -\frac{G_{r}}{q \Omega_{0}\left(P_{r} q^{\alpha}-\Omega_{2}\right) \sinh \sqrt{\overline{P_{r} q^{\alpha}}}} \\
& \times\left(q G(q) \sinh \left((1-\chi) \sqrt{P_{r} q^{\alpha}}\right)-\sinh \left(\chi \sqrt{P_{r} q^{\alpha}}\right)\right) .
\end{aligned}
$$


Let us consider

$$
\bar{w}(\chi, q)=I_{1}(\chi, q)+I_{2}(\chi, q)-I_{3}(\chi, q),
$$

where

$$
\begin{aligned}
I_{1}(\chi, q) & =\frac{F(q)}{\sinh \sqrt{\Omega_{2}}} \sinh \left((1-\chi) \sqrt{\Omega_{2}}\right), \\
& =F(q) \frac{e^{(1-\chi) \sqrt{\Omega_{2}}}-e^{-(1-\chi) \sqrt{\Omega_{2}}}}{e^{\sqrt{\Omega_{2}}}-e^{-\sqrt{\Omega_{2}}}}, \\
& =F(q) \sum_{k=0}^{\infty}\left(e^{-(2 k+\chi) \sqrt{\Omega_{2}}}-e^{-(2 k+1-\chi) \sqrt{\Omega_{2}}}\right),
\end{aligned}
$$

and further simplification of the above expression as

$$
I_{1}(\chi, q)=F(q)(D(\chi, q)-E(\chi, q)) .
$$

The expression for $D(\chi, q)$

$$
\begin{aligned}
D(\chi, q)= & e^{-(2 k+\chi) \sqrt{\Omega_{2}}}, \\
= & \sum_{k=0}^{\infty} \sum_{n=0}^{\infty} \frac{\left(-\Omega_{3}\right)^{n}}{n !} \sum_{m=0}^{\infty} \frac{\Gamma\left(\frac{n}{2}+1\right)}{m ! \Gamma\left(\frac{n}{2}-m+1\right)}\left(\frac{1}{\lambda}\right)^{\frac{n}{2}-m}\left(b-\frac{1}{\lambda}\right)^{m} \\
& \times \sum_{p=0}^{\infty}(-1)^{p} \frac{\Gamma(m+p)}{p ! \Gamma m} \lambda^{p} q^{\alpha p},
\end{aligned}
$$

where $\Omega_{3}=(2 k+\chi) \sqrt{1+\lambda_{1}}$ and $b=\frac{1}{k}+H_{a}^{2}$.

The inverse Laplace transform of the above equation

$$
\begin{aligned}
D(\chi, t)= & e^{-(2 k+\chi) \sqrt{\Omega_{2}}}, \\
= & \sum_{k=0}^{\infty} \sum_{n=0}^{\infty} \frac{\left(-\Omega_{3}\right)^{n}}{n !} \sum_{m=0}^{\infty} \frac{\Gamma\left(\frac{n}{2}+1\right)}{m ! \Gamma\left(\frac{n}{2}-m+1\right)}\left(\frac{1}{\lambda}\right)^{\frac{n}{2}-m}\left(b-\frac{1}{\lambda}\right)^{m} \\
& \times \sum_{p=0}^{\infty}(-1)^{p} \frac{\Gamma(m+p)}{p ! \Gamma m} \lambda^{p} \frac{t^{-\alpha p+1}}{\Gamma(-\alpha p)} .
\end{aligned}
$$

Let us consider now

$$
\begin{aligned}
E(\chi, q)= & e^{-(2 k+\chi) \sqrt{\Omega_{2}}}, \\
= & \sum_{k=0}^{\infty} \sum_{n=0}^{\infty} \frac{\left(-\Omega_{4}\right)^{n}}{n !} \sum_{m=0}^{\infty} \frac{\Gamma\left(\frac{n}{2}+1\right)}{m ! \Gamma\left(\frac{n}{2}-m+1\right)}\left(\frac{1}{\lambda}\right)^{\frac{n}{2}-m}\left(b-\frac{1}{\lambda}\right)^{m} \\
& \times \sum_{p=0}^{\infty}(-1)^{p} \frac{\Gamma(m+p)}{p ! \Gamma m} \lambda^{p} q^{\alpha p},
\end{aligned}
$$

where $\Omega_{4}=(2 k+1-\chi) \sqrt{1+\lambda_{1}}$ and $b=\frac{1}{k}+H_{a}^{2}$. 
Employing the inverse Laplace transform on the above equation gives

$$
\begin{aligned}
E(\chi, t)= & e^{-(2 k+\chi) \sqrt{\Omega_{2}}}, \\
= & \sum_{k=0}^{\infty} \sum_{n=0}^{\infty} \frac{\left(-\Omega_{4}\right)^{n}}{n !} \sum_{m=0}^{\infty} \frac{\Gamma\left(\frac{n}{2}+1\right)}{m ! \Gamma\left(\frac{n}{2}-m+1\right)}\left(\frac{1}{\lambda}\right)^{\frac{n}{2}-m}\left(b-\frac{1}{\lambda}\right)^{m} \\
& \times \sum_{p=0}^{\infty}(-1)^{p} \frac{\Gamma(m+p)}{p ! \Gamma m} \lambda^{p} \frac{t^{-\alpha p+1}}{\Gamma(-\alpha p)} .
\end{aligned}
$$

Keeping in mind Eq. (29) and Eq. (31) while taking the inverse Laplace transform of Eq. (27), we get

$$
I_{1}(\chi, t)=f(t) *(D(\chi, t)-E(\chi, t)) .
$$

Let us consider now

$$
\begin{aligned}
I_{2}(\chi, q)= & \frac{G_{r}}{q \Omega_{0}\left(P_{r} q^{\alpha}-\Omega_{2}\right) \sinh \sqrt{\Omega_{2}}}\left(q G(q) \sinh \left((1-\chi) \sqrt{\Omega_{2}}\right)-\sinh \left(\chi \sqrt{\Omega_{2}}\right)\right), \\
= & \frac{G_{r}\left(1+\lambda_{1}\right)}{\left(P_{r} q^{\alpha}\left(1+\lambda q^{\alpha}\right)-\left(q^{\alpha}+b\right)\left(1+\lambda_{1}\right)\right)} \\
& \times\left(\frac{q G(q) \sinh \left((1-\chi) \sqrt{\Omega_{2}}\right)-\sinh \left(\chi \sqrt{\Omega_{2}}\right.}{q \sinh \sqrt{\Omega_{2}}}\right) .
\end{aligned}
$$

For simplicity, we write the expression of $I_{2}(\chi, q)$

$$
I_{2}(\chi, q)=G_{r}\left(1+\lambda_{1}\right) J(\chi, q)(q G(q) G(\chi, q)-H(\chi, q))
$$

where

$$
J(\chi, q)=\frac{1}{P_{r} q^{\alpha}\left(1+\lambda q^{\alpha}\right)-\left(q^{\alpha}+b\right)\left(1+\lambda_{1}\right)},
$$

and its inverse Laplace transform

$$
J(\chi, t)=-\sum_{k=0}^{\infty} \sum_{n=0}^{\infty} \frac{a_{0}^{k-n}}{c_{0}^{k+1}} \frac{k !}{n !(k-n) !} \frac{t^{\alpha(n-2 k)-1}}{\Gamma \alpha(n-2 k)},
$$

where $a_{0}=P_{r} \lambda, b_{0}=P_{r}+1+\lambda_{1}, c_{0}=b\left(1+\lambda_{1}\right)$.

$$
G(\chi, q)=\frac{\sinh \left((1-\chi) \sqrt{\Omega_{2}}\right)}{q \sinh \sqrt{\Omega_{2}}}
$$

and

$$
H(\chi, q)=\frac{\sinh \left(\chi \sqrt{\Omega_{2}}\right)}{q \sinh \sqrt{\Omega_{2}}} .
$$


The inverse Laplace transform on Eq. (37) and Eq. (38) is transformed into respectively

$$
\begin{aligned}
G(\chi, t)= & \sum_{k=0}^{\infty} \sum_{n=0}^{\infty} \frac{\left(\left(-\Omega_{3}\right)^{n}-\left(-\Omega_{5}\right)^{n}\right)}{n !} \sum_{m=0}^{\infty} \frac{\Gamma\left(\frac{n}{2}+1\right)}{m ! \Gamma\left(\frac{n}{2}-m+1\right)}\left(\frac{1}{\lambda}\right)^{\frac{n}{2}-m}\left(b-\frac{1}{\lambda}\right)^{m} \\
& \times \sum_{p=0}^{\infty}(-1)^{p} \frac{\Gamma(m+p)}{p ! \Gamma m} \lambda^{p} \frac{t^{-\alpha p+1}}{\Gamma(-\alpha p)}
\end{aligned}
$$

where $\Omega_{3}=(2 k+\chi) \sqrt{1+\lambda_{1}}$ and $\Omega_{5}=(2 k-2-\chi) \sqrt{1+\lambda_{1}}$ and

$$
\begin{aligned}
H(\chi, t) & =\sum_{k=0}^{\infty} \sum_{n=0}^{\infty} \frac{\left(\left(-\Omega_{6}\right)^{n}-\left(-\Omega_{4}\right)^{n}\right)}{n !} \sum_{m=0}^{\infty} \frac{\Gamma\left(\frac{n}{2}+1\right)}{m ! \Gamma\left(\frac{n}{2}-m+1\right)}\left(\frac{1}{\lambda}\right)^{\frac{n}{2}-m}\left(b-\frac{1}{\lambda}\right)^{m} \\
& \times \sum_{p=0}^{\infty}(-1)^{p} \frac{\Gamma(m+p)}{p ! \Gamma m} \lambda^{p} \frac{t^{-\alpha p+1}}{\Gamma(-\alpha p)}
\end{aligned}
$$

where $\Omega_{6}=(2 k+1+\chi) \sqrt{1+\lambda_{1}}$ and $\Omega_{4}=(2 k+1-y) \sqrt{1+\lambda_{1}}$.

The $I_{2}(\chi, t)$ is expressed as

$$
I_{2}(\chi, t)=G_{r}\left(1+\lambda_{1}\right) J(\chi, t) *\left(g^{\prime}(t) * G(\chi, t)-H(\chi, t)\right),
$$

where we get the values of $G(\chi, t)$ and $H(\chi, t)$ from Eq. (39) and Eq. (40) respectively.

$$
\begin{aligned}
I_{3}(\chi, t)= & \frac{G_{r}}{q \Omega_{0}\left(P_{r} q^{\alpha}-\Omega_{2}\right) \sinh \sqrt{P_{r} q^{\alpha}}} \\
& \times\left(q G(q) \sinh \left((1-\chi) \sqrt{P_{r} q^{\alpha}}\right)-\sinh \left(\chi \sqrt{P_{r} q^{\alpha}}\right)\right), \\
= & \frac{G_{r}\left(1+\lambda_{1}\right)}{\left(P_{r} q^{\alpha}\left(1+\lambda q^{\alpha}\right)-\left(q^{\alpha}+b\right)\left(1+\lambda_{1}\right)\right)} \\
& \times\left(\frac{q G(q) \sinh \left((1-\chi) \sqrt{P_{r} q^{\alpha}}\right)-\sinh \left(\chi \sqrt{P_{r} q^{\alpha}}\right.}{q \sinh \sqrt{P_{r} q^{\alpha}}}\right) .
\end{aligned}
$$

For simplification,

$$
I_{3}(\chi, q)=G_{r}\left(1+\lambda_{1}\right) J(\chi, q)\left(q G(q) \psi_{1}(\chi, q)-\psi_{2}(\chi, q)\right)
$$

and its inverse Laplace transform gives

$$
I_{3}(\chi, t)=G_{r}\left(1+\lambda_{1}\right) J(\chi, t) *\left(g^{\prime}(t) * \psi_{1}(\chi, t)-\psi_{2}(\chi, t)\right)
$$

where $J(\chi, t), \psi_{1}(\chi, t), \psi_{2}(\chi, t)$ are attained in Eqs. (36), (17), and (19) respectively.

By implementing the inverse Laplace transform on Eq. (25), bearing in mind the expression of $I_{1}(\chi, t), I_{2}(\chi, t), I_{3}(\chi, t)$ from Eq. (32), Eq. (41), and Eq. (44) respectively, we will get the velocity solution as

$$
w(\chi, t)=I_{1}(\chi, t)+I_{2}(\chi, t)-I_{3}(\chi, t) .
$$




\section{Limiting cases}

\subsection{Solution of velocity when $\lambda_{1} \rightarrow 0$}

By taking $\lambda_{1} \rightarrow 0$, the solution obtained for velocity profile in Eq. (24) changes into the solution of second grade fluid

$$
\begin{aligned}
\bar{w}(\chi, q)= & \frac{F(q)}{\sinh \sqrt{\Xi_{2}}} \sinh \left((1-\chi) \sqrt{\Xi_{2}}\right) \\
& +\frac{G_{r}}{q \Xi_{0}\left(P_{r} q^{\alpha}-\Xi_{2}\right) \sinh \sqrt{\Xi_{2}}}\left(q G(q) \sinh \left((1-\chi) \sqrt{\Xi_{2}}\right)-\sinh \left(\chi \sqrt{\Xi_{2}}\right)\right) \\
& -\frac{G_{r}}{q \Xi_{0}\left(P_{r} q^{\alpha}-\Xi_{2}\right) \sinh \sqrt{P_{r} q^{\alpha}}} \\
& \times\left(q G(q) \sinh \left((1-\chi) \sqrt{\overline{P_{r} q^{\alpha}}}\right)-\sinh \left(\chi \sqrt{\overline{P_{r} q^{\alpha}}}\right)\right),
\end{aligned}
$$

where $\Xi_{0}=1+\lambda q^{\alpha}, \Xi_{2}=\frac{\Omega_{1}}{\Xi_{0}}$. For $\alpha \rightarrow 1$, we will get the limiting result obtained by [46].

\subsection{Solution of velocity when $\lambda \rightarrow 0, \lambda_{1} \rightarrow 0$}

By choosing $\lambda \rightarrow 0, \lambda_{1} \rightarrow 0$, and $\frac{1}{K}=0$ (absence of porosity), the results obtained for velocity reduce to viscous fluid

$$
\begin{aligned}
\bar{w}(\chi, q)= & \frac{F(q)}{\sinh \sqrt{q^{\alpha}+H_{a}^{2}}} \sinh \left((1-\chi) \sqrt{q^{\alpha}+H_{a}^{2}}\right) \\
& +\frac{G_{r}}{q\left(P_{r} q^{\alpha}-\left(q^{\alpha}+H_{a}^{2}\right)\right) \sinh \sqrt{q^{\alpha}+H_{a}^{2}}} \\
& \times\left(q G(q) \sinh \left((1-\chi) \sqrt{q^{\alpha}+H_{a}^{2}}\right)-\sinh \left(\chi \sqrt{q^{\alpha}+H_{a}^{2}}\right)\right) \\
& -\frac{G_{r}}{q\left(P_{r} q^{\alpha}-\left(q^{\alpha}+H_{a}^{2}\right)\right) \sinh \sqrt{P_{r} q^{\alpha}}} \\
& \times\left(q G(q) \sinh \left((1-\chi) \sqrt{P_{r} q^{\alpha}}\right)-\sinh \left(\chi \sqrt{P_{r} q^{\alpha}}\right)\right) .
\end{aligned}
$$

For $\alpha \rightarrow 1$, we will get the result for viscous fluid as obtained by [42] $\left(G_{m}=0\right)$. For exact solutions of the limiting cases, we can follow the same steps as the ones used to find the exact solutions of Jeffery fluid.

\section{Graphical illustration and discussion}

The study of transference of heat of time-dependent MHD natural convection flow of Jeffery fluid via Caputo fractional derivative operator is elaborated here. The solution for temperature and velocity is explored with the Laplace transform. The obtained exact solution satisfies all the imposed initial and generalized boundary conditions. The functions $f(t)$ and $g(t)$ are assumed constant for the velocity and temperature field. To get a perception of the physical significance of the associated parameters for the fluid flow, the assistance of a graph is taken. The physical parameter impacts such as relaxation time $\lambda$, Grashof number $G_{r}$, Prandtl number $P_{r}$, permeability parameter $K$, Jeffery fluid parameter $\lambda_{1}$, and $\alpha$ (memory parameter) on the temperature and velocity profile are illustrated here. 


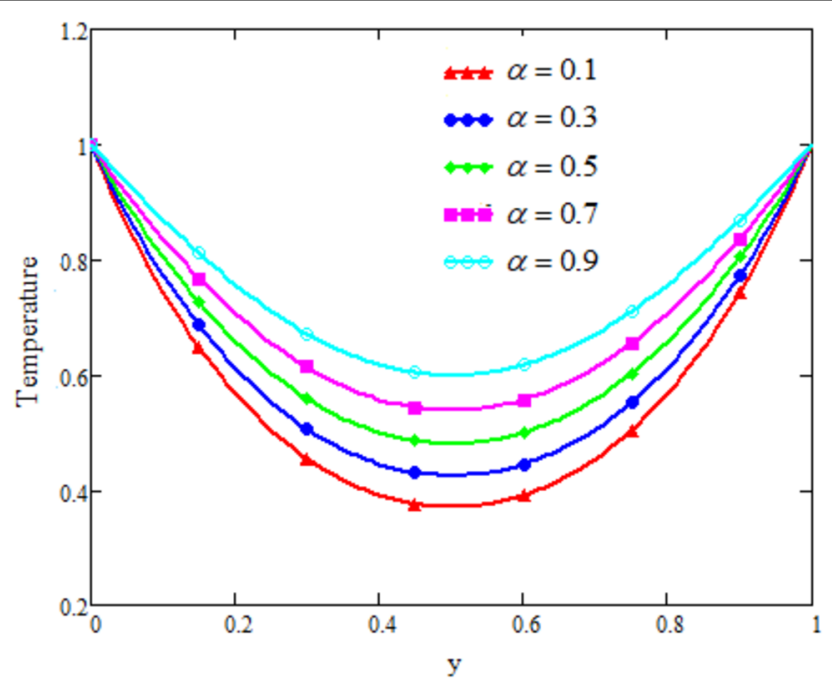

Figure 2 Temperature curves along $\alpha$ variation and $P_{r}=12, t=1.5$

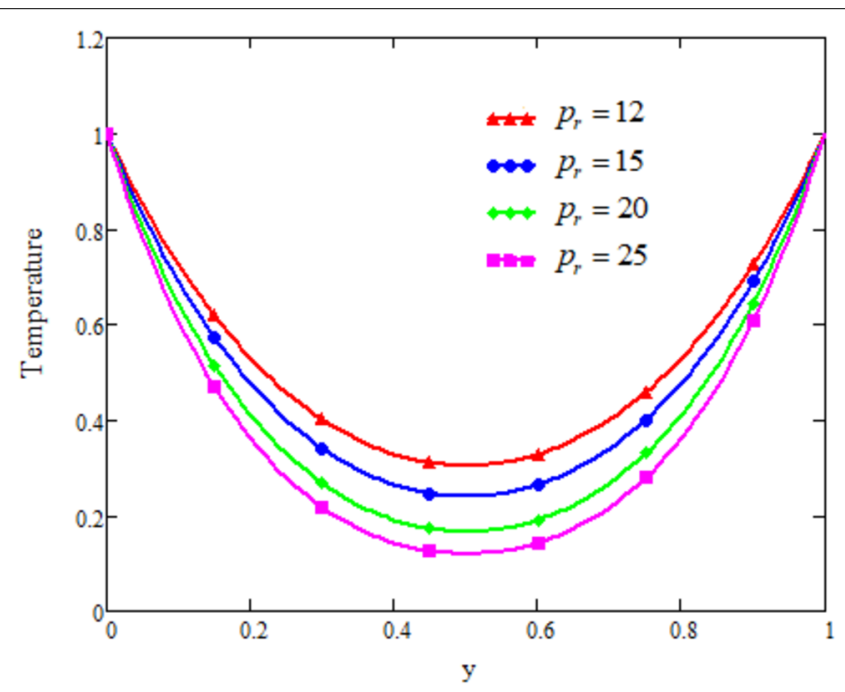

Figure 3 Temperature curves along $P_{r}$ variation and $\alpha=0.5, t=1.5$

The fractional parameter $\alpha$ control on the temperature profile is presented in Fig. 2. As $\alpha$ varies from small to large, we see the temperature become the increasing function of $\alpha$. As $\alpha$ increases, boundary layer thickness increase results in elevating the temperature. It is easy to validate the result for $\alpha \rightarrow 1$, which are already present in the literature [46].

The Prandtl number controls the thickness of the thermal boundary layer. The increase in $P_{r}$ dominates the momentum diffusivity over the fluid flow, which causes the decline in heat diffusion rate. So $P_{r}$ elevates, reduces the thermal boundary layer thickness which drops the temperature profile as sketched in Fig. 3.

The governing of time on the temperature profile is plotted in Fig. 4. It has come into notice that the temperature elevates with the increase in time. The spatial variable increase results in an expansion in the thermal boundary layer, and hence temperature escalates. 


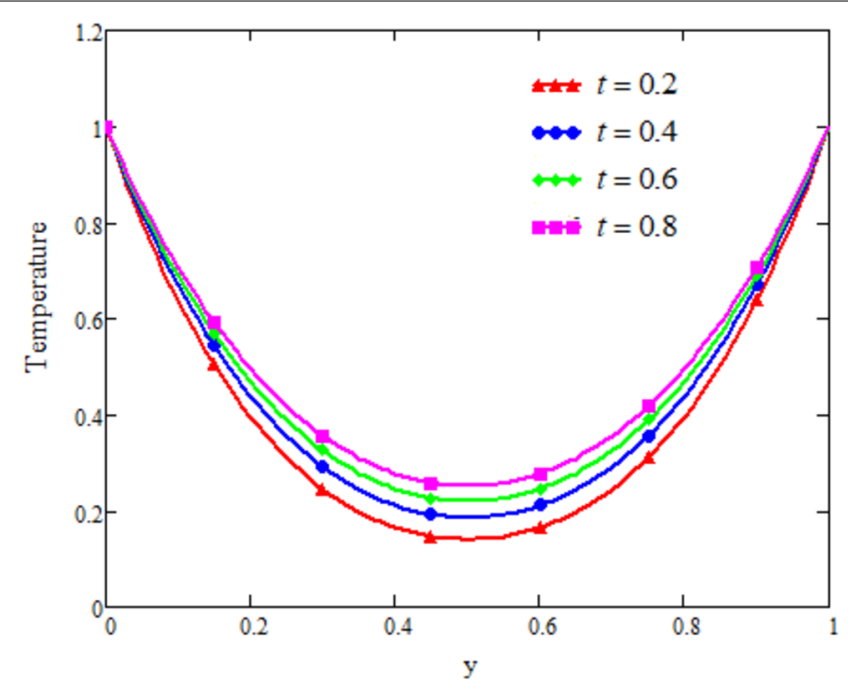

Figure 4 Temperature curves along $t$ variation and $P_{r}=12, \alpha=0.5$

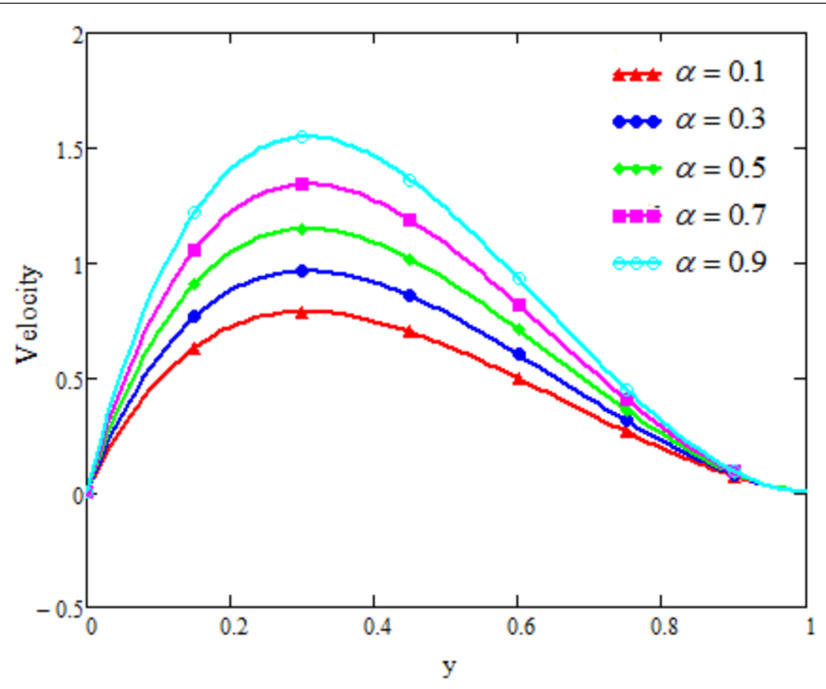

Figure 5 Velocity curves along $\alpha$ variation and $P_{r}=15, G_{r}=15.5, K=12.5, \lambda=1.9, \lambda_{1}=1.6, H_{a}=0.8, t=5$

The governing of memory parameter $\alpha$ on the fluid flow is shown in Fig. 5. It is evident that with the rise in $\alpha$, fluid flow accelerates. The reason is $\alpha$ increases, boundary layer thickness increases, resulting in acceleration of the velocity.

To elaborate on the impacts of the Prandtl number on the flow of the fluid, Fig. 6 is sketched. Adding up in the value of $P_{r}$, the fluid becomes thicker, and the thickness of the boundary layer is reduced. Consequently, this causes a slow down in the speed of fluid flow.

In Fig. 7, the impact of $G_{r}$ on the velocity field is depicted. We noticed the fluid accelerates with greater velocity with higher values of thermal Grashof number $G_{r}$. Since the Grashof number is a ratio of buoyancy forces to viscous forces, it is evident that enhancement in $G_{r}$ results in the dominance of buoyancy forces rather than viscous forces, which causes the natural convection and accelerates the velocity. 


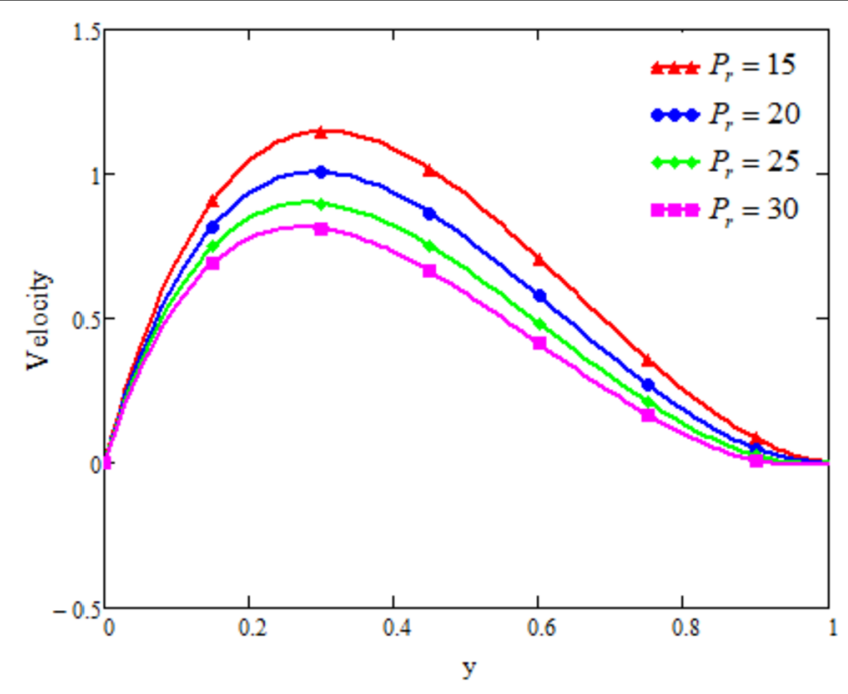

Figure 6 Velocity curves along $P_{r}$ variation and $\alpha=0.5, G_{r}=15.5, K=12.5, \lambda=1.9, \lambda_{1}=1.6, H_{a}=0.8, t=5$

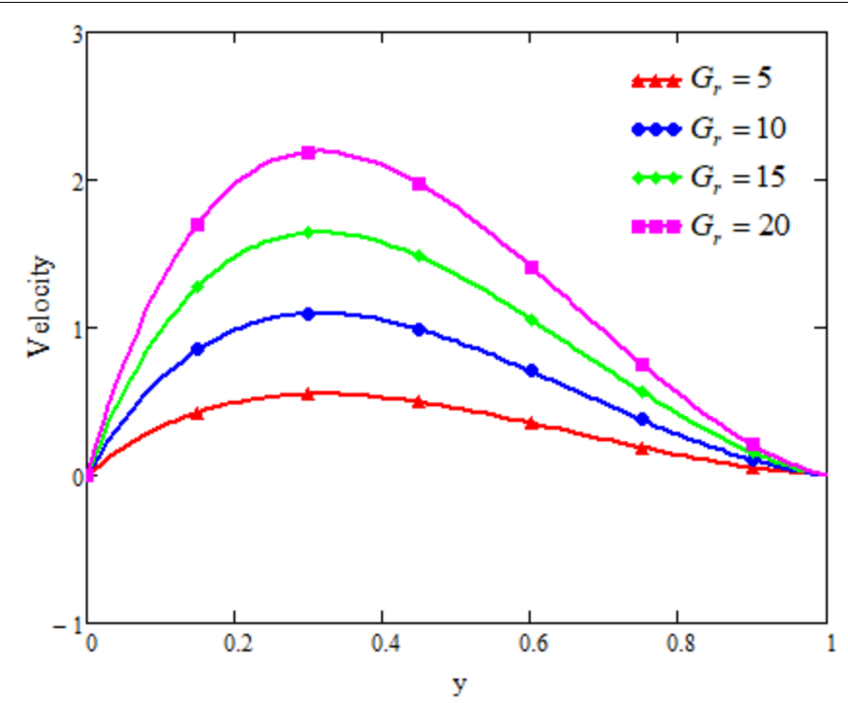

Figure 7 Velocity curves along $G_{r}$ variation and $\alpha=0.5, P_{r}=11.5, K=3.5, \lambda=0.4, \lambda_{1}=1.6, H_{a}=0.8, t=5$

By keeping the other parameters involved fixed and changing the value of the porosity parameter on the velocity field, a plot is drawn in Fig. 8. It has come to our attention that the fluid velocity drops down with the rise in the value of $K$. The resistive force due to the porous medium is the major reason for the fall of the fluid velocity.

Figure 9 shows the influence of the Hartmann number on the velocity of the fluid. The rise in Hartmann's number depreciates the speed of the fluid flow. Physically, Lorentz's force (resistive force) opposes the fluid motion, which results in the slow down of fluid flow.

The influence of relaxation time on the fluid velocity is sketched in Fig. 10. It is noticed that increasing the relaxation time $\lambda$, the velocity curves decrease. The relaxation time increase results in the expansion of the boundary layer, which causes the velocity to fall. 


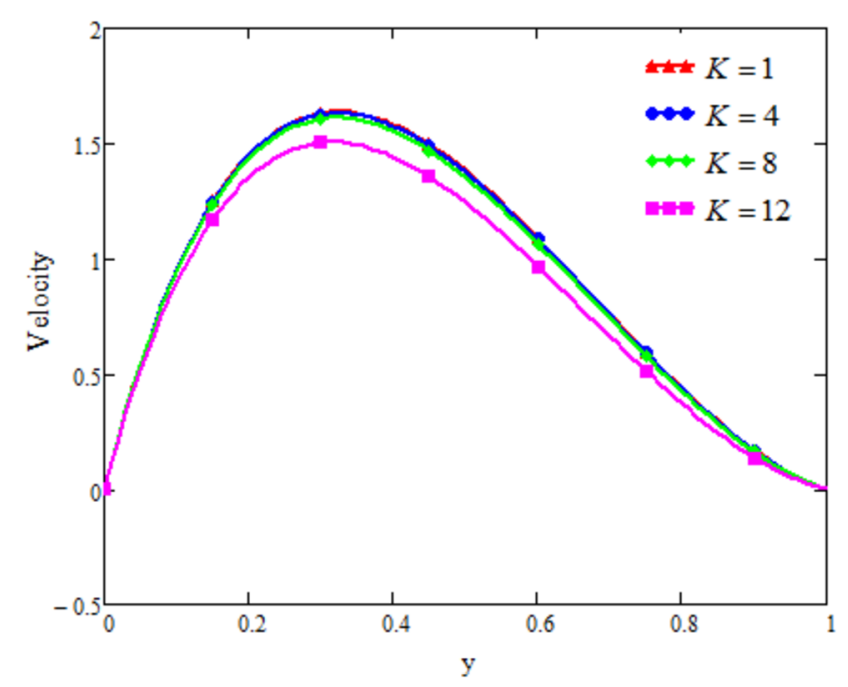

Figure 8 Velocity curves along $K$ variation and $\alpha=0.5, P_{r}=1.5, G_{r}=5.5, H_{a}=0.3, \lambda=0.6, \lambda_{1}=1.9, t=5$

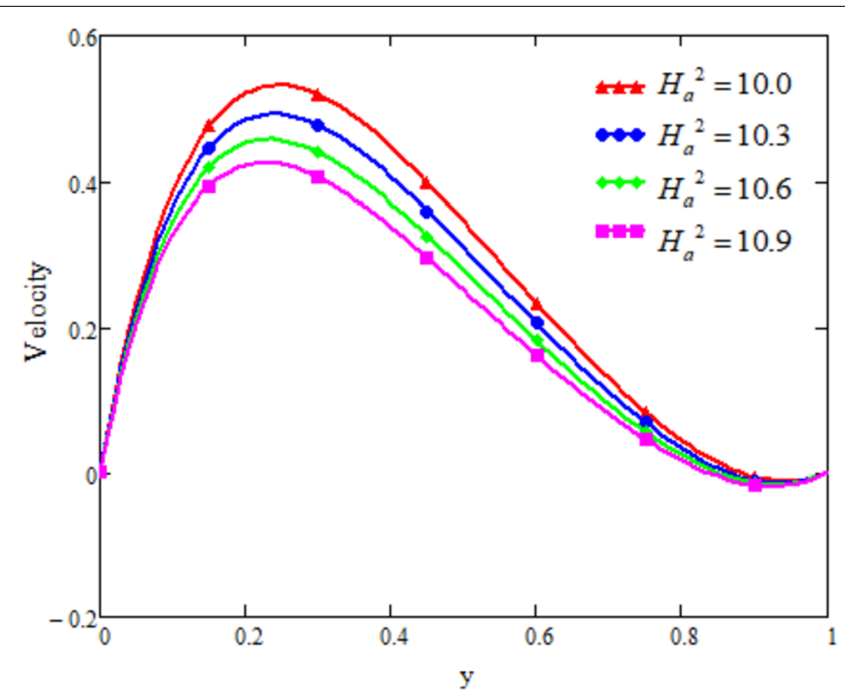

Figure 9 Velocity curves along $H_{a}$ variation and $\alpha=0.5, P_{r}=11.5, G_{r}=8, K=1.5, \lambda=0.4, \lambda_{1}=1.6, t=5$

To visualize the effects of Jeffery fluid parameter $\lambda_{1}$ on the velocity profile, Fig. 11 is plotted. We observed the enhancement in the momentum boundary layer thickness owing to an increase in $\lambda_{1}$ which increases fluid flow.

Figure 12 focuses on the impact of time on the Jeffery fluid flow. It has been observed that an increase in time drops the velocity of the fluid. From the physical point of view, the boundary layer thickness gets minimized over time.

\section{Closing remarks}

The study of MHD Jeffery fluid on two parallel vertical plates dipped in a permeable medium is elaborated here via fractional Caputo approach. The exact expressions for temperature and velocity are attained by the Laplace integral transform. The impacts of associated parameters for the fluid flows are discussed. The main observations are the following: 


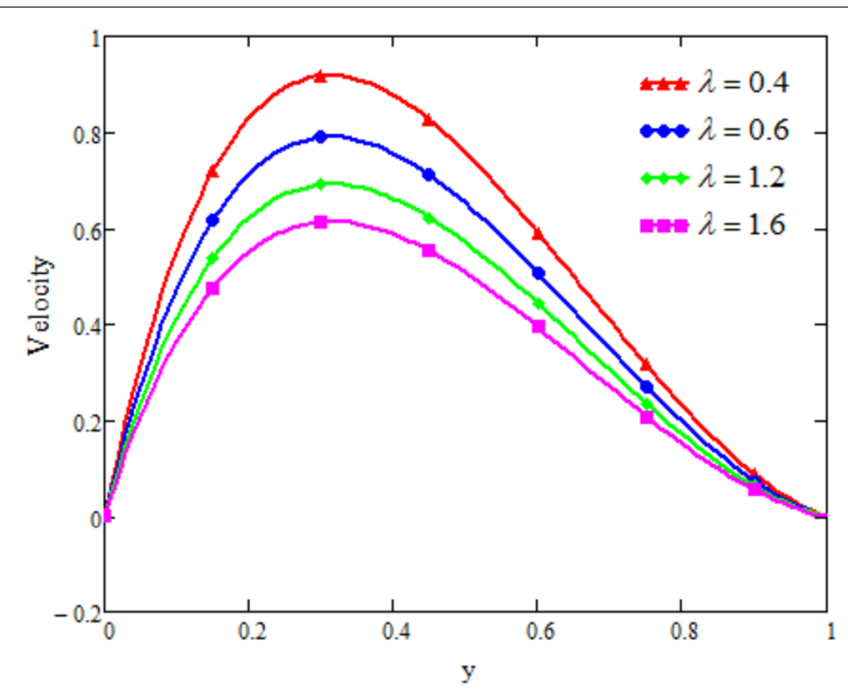

Figure 10 Velocity curves along $\lambda$ variation and $\alpha=0.5, P_{r}=11.5, G_{r}=6.5 K=2, \lambda_{1}=1.8, H_{a}=0.6, t=5$

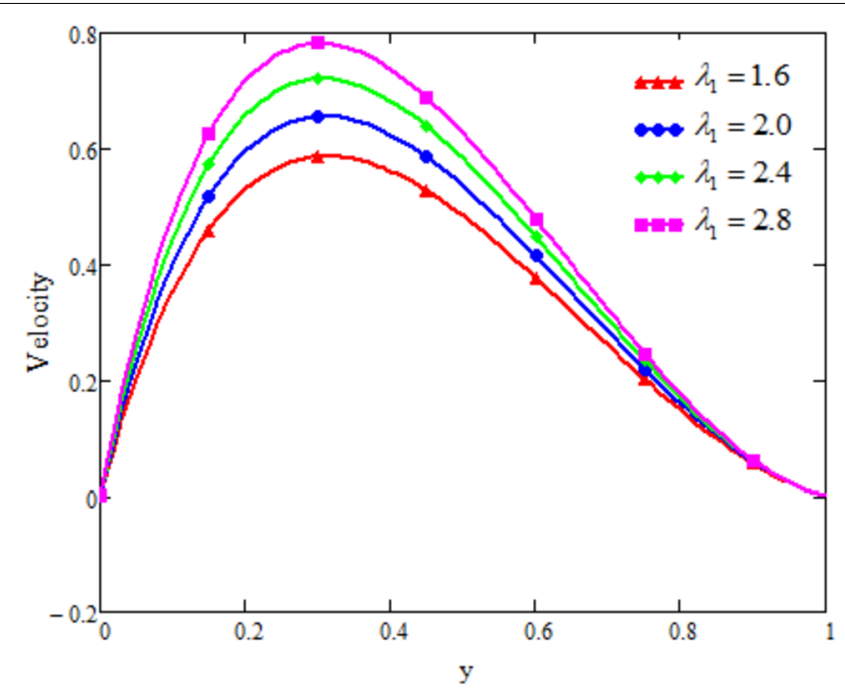

Figure 11 Velocity curves along $\lambda_{1}$ variation and $\alpha=0.5, P_{r}=11.5, G_{r}=4.5, K=1.5, \lambda=0.4, H_{a}=0.6, t=5$

- The increase in the value of fractional parameter $\alpha$ shows an increase in the boundary layer thickness, which increases velocity and temperature.

- The decay or slow down is observed in fluid velocity with the rise in $P_{r}, \lambda, H_{a}$, and K.

- It is noticed that the fluid velocity escalates with elevation in the values of $G_{r}$ and $\lambda_{1}$.

- Temperature profile declines due to the strong influence in the expansion in the values of $P_{r}$ and time.

- In a limiting case, when $\alpha \rightarrow 1$, the results recovered are of integer-order derivative Jeffery fluid.

- For both $\lambda \rightarrow 0, \lambda_{1} \rightarrow 0$ and $\alpha \rightarrow 1$, the results obtained are for viscous fluid.

- These results can be evaluated with other fractional derivative approaches, and useful comparison can be drawn between the various fractional approaches. 


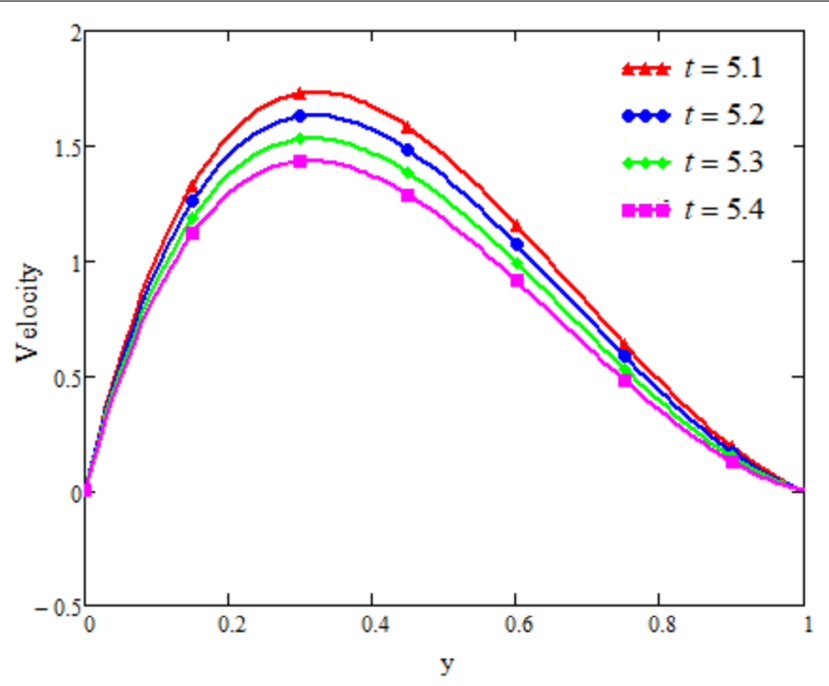

Figure 12 Velocity curves along $t$ variation and $\alpha=0.5, P_{r}=15, G_{r}=15.5, K=12.5, \lambda=1.9, \lambda_{1}=1.6, H_{a}=0.8$, $t=5$

\section{Acknowledgements}

This research was supported by Taif University Researchers Supporting Project Number (TURSP-2020/96), Taif University, Taif, Saudi Arabia. The authors are also grateful to anonymous referees for their valuable suggestions which significantly improved this manuscript.

\section{Funding}

No external funding is available for this research.

\section{Abbreviations}

$w$, Fluid velocity; $B_{0}$, Applied magnetic field; $q$, Laplace transform parameter; $\theta$, Fluid temperature; $k$, Heat conductivity; $\rho$, Fluid density; $\lambda$, Relaxation time; $\sigma$, Electric conductivity; $\mu$, Dynamic viscosity of fluid; $v$, Kinematic viscosity of fluid; $c_{p}$, Specific heat; $g$, Gravitational acceleration; $H_{a}^{2}$, Hartmann number; $P_{r}$, Prandtl number; $G_{r}$, Thermal Grashof number; $K$, Porosity parameter; $\lambda_{1}$, Jeffery fluid parameter.

\section{Availability of data and materials}

Not applicable.

\section{Competing interests}

The authors declare that they have no competing interests.

\section{Authors' contributions}

All authors equally contributed to this work. All authors read and approved the final manuscript.

\section{Author details}

'Department of Mathematics, Government College University, 54000 Lahore, Pakistan. ${ }^{2}$ Department of Mathematics and Statistics, College of Science, Taif University, 21944 Taif, Saudi Arabia. ${ }^{3}$ Department of Mathematics, University of Management and Technology, 40100 C-II Johar Town Lahore, Pakistan. ${ }^{4}$ Institute for Groundwater Studies (IGS), University of the Free State, 9300 Bloemfontein, South Africa. ${ }^{5}$ Department of Mathematics, University of Sargodha, 40100 Sargodha, Pakistan.

\section{Publisher's Note}

Springer Nature remains neutral with regard to jurisdictional claims in published maps and institutional affiliations.

Received: 9 May 2021 Accepted: 29 July 2021 Published online: 19 August 2021

\section{References}

1. Fetecau, C., Jamil, M., Fetecau, C., Siddique, I.: A note on the second problem of Stokes for Maxwell fluids. Int. J. Non-Linear Mech. 44, 1085-1090 (2009)

2. Vieru, D., Fetecau, C., Fetecau, C.: Flow of a viscoelastic fluid with fractional Maxwell model between two side walls perpendicular to a plate. Appl. Math. Comput. 200, 459-464 (2008)

3. Vieru, D., Rauf, A.: Stokes flows of a Maxwell fluid with wall slip condition. Can. J. Phys. 89(10), 1061-1071 (2012)

4. Dunn, J.E., Rajagopal, K.R.: Fluid of differential type: critical review and thermodynamic analysis. Int. J. Eng. Sci. 33, 689-729 (1995) 
5. Rajagopal, K.R.: Mechanics of non-Newtonian fluids. In: Galdi, G.P., Necas, J. (eds.) Recent Developments in Theoretical Fluid Mechanics. Pitman Res. Notes in Math. Ser., vol. 291. Longman, New York (1993)

6. Hussain, T., Shehzad, S.A., Hayat, T., Alsaedi, A., Al-Solamy, F., Ramzan, M.: Radiative hydromagnetic flow of Jeffrey nanofluid by an exponentially stretching sheet. PLoS ONE 9, 1-9 (2014)

7. Hayat, T., Mustafa, M.: Influence of thermal radiation on the unsteady mixed convection flow of a Jeffery fluid over a stretching sheet. Z. Naturforsch. 65a, 711-719 (2010)

8. Idowu, A.S., Jimoh, A., Ahmed, L.O.: Impact of heat and mass transfer on MHD oscillatory flow of Jeffery fluid in a porous channel with thermal conductivity, Dufour and Soret. J. Appl. Sci. Environ. Manag. 19(4), 819-830 (2015)

9. Zin, N.A.M., Khan, I., Shafie, S.: Influence of thermal radiation on unsteady MHD free convection flow of Jeffrey fluid over a vertical plate with ramped wall temperature. Math. Probl. Eng. 2016, 6257071 (2016)

10. Zeeshan, A., Majeed, A.: Heat transfer analysis of Jeffery fluid flow over a stretching sheet with suction/injection and magnetic dipole effect. Alex. Eng. J. 55(3), 2171-2181 (2016)

11. Bhatti, M.M., Zeeshan, A.: Analytic study of heat transfer with variable viscosity on solid particle motion in dusty Jeffery fluid. Mod. Phys. Lett. B 30(16), 1650196 (2016)

12. Turkyilmazoglu, M.: Unsteady convection flow of some nanofluids past a moving vertical flat plate with heat transfer. J. Heat Transf. 136(3), 031704 (2014)

13. Pourabdian, M., Qate, M., Morad, M.R., Javareshkian, A.R.: The Jeffery-Hamel Flow and Heat Transfer of Nanofluids by Homotopy Perturbation Method and Comparison with Numerical Results. The 13th Iranian Aerospace Soci. Conf. (2014)

14. Kothandapani, M., Prakash, J.: Convective boundary conditions effect on peristaltic flow of a MHD Jeffery nanofluid. Appl. Nanosci. 6(3), 323-335 (2016)

15. Saqib, M., Ali, F., Khan, I., Sheikh, N.A.: Heat and mass transfer phenomena in the flow of Casson fluid over an infinite oscillating plate in the presence of first-order chemical reaction and slip effect. Neural Comput. Appl. 30(7), 2159-2172 (2018)

16. Gaffar, S.A., Prasad, V.R., Reddy, E.K.: Computational study of Jeffrey's non-Newtonian fluid past a semi-infinite vertical plate with thermal radiation and heat generation/absorption. Ain Shams Eng. J. 8(2), 277-294 (2017)

17. Agrawal, P., Dadheech, P.K., Nisar, K.S., Purohit, S.D.: Magneto Marangoni flow of $\mathrm{Gama}-\mathrm{Al}_{2} \mathrm{O}_{3}$ nanofluids with thermal radiation and heat source/sink effects over a stretching surface embedded in porous medium. Case Stud. Therm. Eng. 23, 100802 (2021)

18. Mathur, P., Mishra, S.R., Purohit, S.D., Bohra, M.: Entropy generation in a micropolar fluid past an inclined channel with velocity slip and heal flux conditions: variation parameters method. Heat Transf (2021). https://doi.org/10.1002/htj.22236

19. Kirtika, A.R., Purohit, S.D.: Mathematical model for anomalous subdiffusion using conformable operator. Chaos Solitons Fractals 140, 110199 (2020)

20. Shahid, N.: A study of heat and mass transfer in a fractional MHD flow over an infinite oscillating plate. SpringerPlus 4(1), 640 (2015)

21. Vieru, D., Fetecau, C., Fetecau, C.: Time-fractional free convection flow near a vertical plate with Newtonian heating and mass diffusion. Therm. Sci. 19(1), S85-S98 (2015)

22. Khan, I., Shah, N.A., Vieru, D.: Unsteady flow of generalized Casson fluid with fractional derivative due to an infinite plate. Eur. Phys. J. Plus 131(6), 1-12 (2016)

23. Riaz, M.B., Siddiqui, I., Saeed, S.T., Atangana, A.: MHD Oldroyd-B fluid with slip condition in view of local and nonlocal kernels. J. Appl. Comput. Mech. 7(1), 116-127 (2021)

24. Khan, M.: Partial slip effects on the oscillatory flows of a fractional Jeffery fluid in a porous medium. J. Porous Media 10, 473-487 (2007)

25. Khan, I.: A note on exact solutions for the unsteady free convection flow of a Jeffrey fluid. Z. Naturforsch. 70, 1-5 (2015)

26. Kumar, S.: A new analytical modelling for fractional telegraph equation via Laplace transform. Appl. Math. Model. 38(13), 3154-3163 (2014)

27. Iftikhar, N., Baleanu, D., Riaz, M.B., Husnine, S.M.: Heat and mass transfer of natural convective flow with slanted magnetic field via fractional operators. J. Appl. Comput. Mech. 7(1), 189-212 (2021)

28. Ghanbari, B., Kumar, S., Kumar, R.: A study of behaviour for immune and tumor cells in immunogenetic tumour model with non-singular fractional derivative. Chaos Solitons Fractals 133, 109619 (2020)

29. Goufo, E.F.D., Kumar, S., Mugisha, S.B.: Similarities in a fifth-order evolution equation with and with no singular kernel. Chaos Solitons Fractals 130, 109467 (2020)

30. Kumar, S., Kumar, R., Agarwal, R.P., Samet, B.: A study of fractional Lotka-Volterra population model using Haar wavelet and Adams-Bashforth-Moulton methods. Math. Methods Appl. Sci. 43(8), 5564-5578 (2020)

31. Kumar, S., Ghosh, S., Samet, B., Goufo, E.F.D.: An analysis for heat equations arises in diffusion process using new Yang-Abdel-Aty-Cattani fractional operator. Math. Methods Appl. Sci. 43(9), 6062-6080 (2020)

32. Kumar, S., Chauhan, R.P., Momani, S., Hadid, S.: Numerical investigations on COVID-19 model through singular and non-singular fractional operators. Numer. Methods Partial Differ. Equ. (2020). https://doi.org/10.1002/num.22707

33. Kumar, S., Kumar, R., Momani, S., Hadid, S.: A study on fractional COVID-19 disease model by using Hermite wavelets. Math. Methods Appl. Sci. (2021). https://doi.org/10.1002/mma.7065

34. Veeresha, P., Prakash, D.G., Kumar, S.: A fractional model for propagation of classical optical solitons by using nonsingular derivative. Math. Methods Appl. Sci. (2020). https://doi.org/10.1002/mma.6335

35. Kumar, S., Kumar, R., Cattani, C., Samet, B.: Chaotic behaviour of fractional predator-prey dynamical system. Chaos Solitons Fractals 135, 109811 (2020)

36. Kumar, S., Ahmadian, A., Kumar, R., Kumar, D.: An efficient numerical method for fractional SIR epidemic model of infectious disease by using Bernstein wavelets. Mathematics 8(4), 558 (2020)

37. Shehzad, S.A., Hayat, T., Alhuthali, M.S., Asghar, S.: MHD three-dimensional flow of Jeffrey fluid with Newtonian heating. J. Cent. South Univ. 21, 1428-1433 (2014)

38. Zafar, A.A., Awrejcewicz, J., Mazur, O., Riaz, M.B.: Study of composite fractional relaxation differential equation using fractional operators with and without singular kernels and special functions. Adv. Differ. Equ. 2021, 87 (2021) 
39. Iftikhar, N., Saeed, S.T., Riaz, M.B.: Fractional study on heat and mass transfer of MHD Oldroyd-B fluid with ramped velocity and temperature. Comput. Methods Differ. Equ. 2021, 1-28 (2021)

40. Hayat, T., Sajjad, R., Asghar, S.: Series solution for MHD channel flow of a Jeffery fluid. Commun. Nonlinear Sci. Numer. Simul. 15(9), 2400-2406 (2010)

41. Iftikhar, N., Baleanu, D., Husnine, S.M., Shabbir, K.: Magnetohydrodynamic mixed convection flow of Jeffery fluid with thermophoresis, Soret and Dufour effects and convective condition. AIP Adv. 9(3), 035251 (2019)

42. Imran, M.A., Aleem, M., Chowdhury, M.S.R., Hussnain, A.: Analysis of mathematical model of fractional viscous fluid through a vertical rectangular channel. Chin. J. Phys. 61, 336-350 (2019)

43. Mainardi, F.: Fractional Calculus and Waves in Linear Viscoelasticity: An Introduction to Mathematical Models. Imperial College Press, London (2010)

44. Mathai, A.M., Saxena, R.K., Haubold, H.J.: The H-Functions: Theory and Applications. Springer, New York (2010)

45. Podlubny, l.: Fractional Differential Equations. Academic Press, San Diego (1999)

46. Aleem, M., Asjad, M.A., Ahmadian, A., Salimi, M., Ferrara, M.: Heat transfer analysis of channel flow of MHD Jeffrey fluid subject to generalized boundary conditions. Eur. Phys. J. Plus 135, 26 (2020)

47. Caputo, M., Fabrizio, M.: A new definition of fractional derivative without singular kernel. Prog. Fract. Differ. Appl. 1(2), $73-85(2015)$

\section{Submit your manuscript to a SpringerOpen ${ }^{\circ}$ journal and benefit from:}

- Convenient online submission

- Rigorous peer review

- Open access: articles freely available online

- High visibility within the field

- Retaining the copyright to your article 